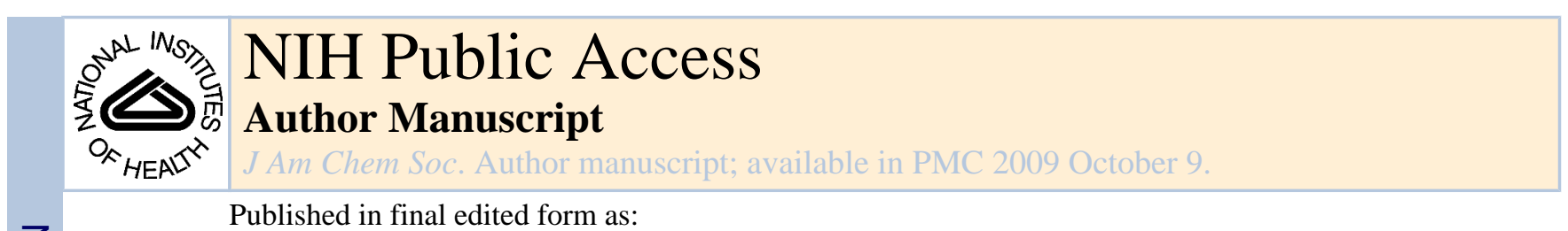

J Am Chem Soc. 2007 May 16; 129(19): 6064-6065. doi:10.1021/ja0704611.

\title{
Nanoparticle Self-Assembly Gated by Logical Proteolytic Triggers
}

\author{
Geoffrey von Maltzahn ${ }^{1}$, Todd J Harris ${ }^{1}$, Ji-Ho Park ${ }^{2}$, Dal-Hee Min ${ }^{1}$, Alexander J Schmidt ${ }^{1}$, \\ Michael J. Sailor ${ }^{2}$, and Sangeeta N. Bhatia ${ }^{1,3,4}$ \\ Geoffrey von Maltzahn: ; Todd J Harris: ; Ji-Ho Park: ; Dal-Hee Min: ; Alexander J Schmidt: ; Michael J. Sailor: ; Sangeeta \\ N. Bhatia: sbhatia@mit.edu \\ ${ }^{1}$ Harvard-MIT Division of Health Sciences and Technology, Cambridge, MA \\ ${ }^{2}$ Department of Chemistry and Biochemistry, University of Californiia, San Diego \\ ${ }^{3} \mathrm{MIT}$ Electrical Engineering and Computer Science \\ ${ }^{4}$ MIT/Brigham \& Women's Hospital, Boston, MA
}

\section{Abstract}

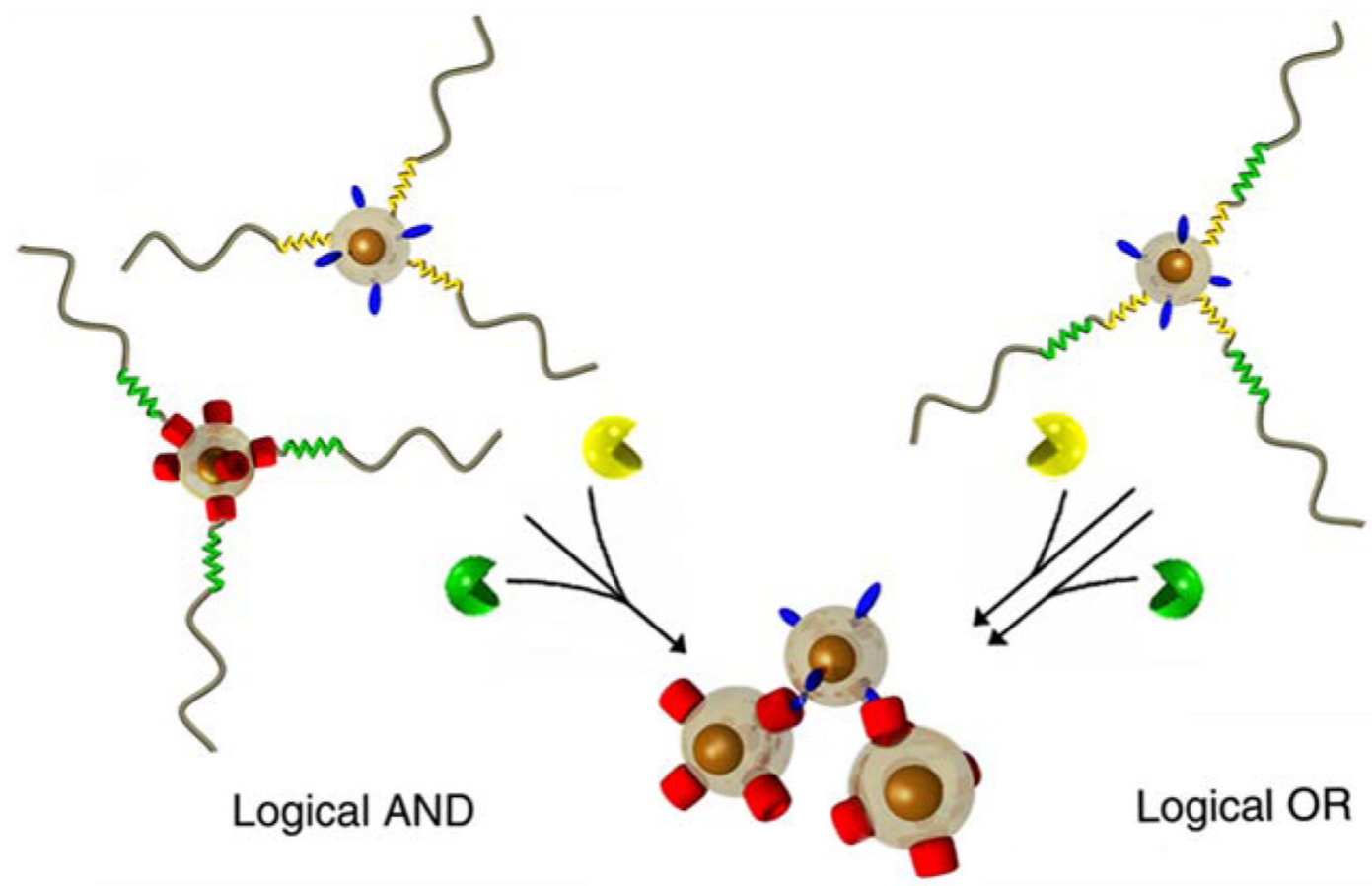

The emergent electromagnetic properties of nanoparticle self-assemblies are being harnessed to build new medical and biochemical assays with unprecedented sensitivity. While current self-assembly assays have displayed superior sensitivity for single molecular targets, development of systems with the capacity to process multiple inputs may more effectively decipher complex disease signatures such as cancer. Herein, we present the design and synthesis of nanoparticles that utilize Boolean logic to simultaneously probe for two proteolytic inputs associated with unique aspects of tumorigenesis (MMP2 and MMP7). Using dynamic light scatting, fluorescence, and MRI, we show that logical AND and OR functions can control the self-assembly of disperse superparamagnetic nanoparticles and enable remote, NMR detection of nanoparticle computation. In the future, by increasing the complexity of assembly triggers, nanoparticles may be tailored to sense a diversity of disease inputs in vitro and potentially in vivo. 
The emergent electromagnetic properties of nanoparticle self-assemblies are being harnessed to build new medical and biochemical assays with unprecedented sensitivity. Nanoparticle (NP) assembly has been exploited to probe for a host of pathological inputs in vitro, including

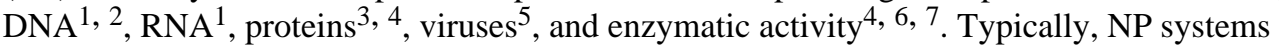
are designed to sense single molecular targets. While this methodology has been effective for in vitro applications, the future development of highly diagnostic in vivo sensors may benefit from the ability to monitor multiple aspects of disease. In this report we describe a method whereby inorganic nanocrystals may utilize Boolean logic to simultaneously process two inputs associated with cancer invasion (MMP2 and MMP7). Disperse, superparamagnetic $\mathrm{Fe}_{3} \mathrm{O}_{4} \mathrm{NPs}$ are designed to coalesce in response to logical AND or OR functions. In either system, NP self-assembly amplifies the T2 relaxation rate of hydrogen protons, enabling remote, MRIbased detection of logical function. In the future, we believe that these sensors may be optimized to monitor a diversity of inputs in vitro and in vivo.

Logical operations were designed to analyze inputs of two matrix-metalloproteinases, a family of at least 26 members of secreted and membrane bound proteases that have been studied extensively for their role in cancer ${ }^{8}$. In particular, matrix-metalloproteinase-2 (MMP2), is overexpressed in many cancers, including breast cancers, and is an indicator of cancer invasiveness, metastasis, angiogenesis, and treatment efficacy ${ }^{9}$. MMP7, a protease with broader substrate specificity, is thought to facilitate early stages of mammary carcinoma progression ${ }^{10}$. In tissues excised from breast cancer patients, both MMP-2 and MMP-7 were expressed at statistically higher levels in carcinogenic than in normal breast tissues ${ }^{11}$, highlighting their potential utility as dual markers of neoplastic inception. We demonstrate using dynamic light scattering (DLS), fluorescence, and MRI that logical sensors can probe samples for the presence of both MMP2 and MMP7 (AND function) or for the presence of either MMP2 or MMP7 (OR function).

To synthesize both sensor types, two kinds of NPs were initially engineered: one with a tethered ligand (biotin) and the other with its receptor (neutravidin). These particles were stable separately, but aggregated readily when combined. We sought to completely mask these groups by attachment of peptide-polyethyleneglycol (PEG) conjugates to conditionally prevent assembly. Previously, we demonstrated that two 10kDa PEG-modified NPs could mutually deter each other's binding ${ }^{6}$. Here, by extending the polymer length to $20 \mathrm{kDa}$, we demonstrate that modification of only one NP can completely inhibit the binding of an unmodified NP (Figure S2; Figure 2). Accordingly, we reasoned that by linking polymers to each particle via unique protease substrates, assembly could be restricted to occur only in the presence of both enzymes (Logical AND) (Scheme 1). Furthermore, by anchoring polymers to only the ligand NP with a tandem peptide substrate (containing both enzyme cleavage motifs in series), we sought to actuate assembly in the presence of either or both of the enzyme inputs (Logical OR) (Scheme 1).

Peptide PEG polymers were synthesized by reacting the peptide $\mathrm{N}$-terminus (or lysine residue for OR tandem peptide) with amine-reactive 20kDa methoxy-PEG-succimidyl $\alpha$ -

methylbutanoate (see supplemental methods for details). Cysteine residues were incorporated at the C-terminus of peptides to allow oriented attachment of substrate polymers to NPs and TAMRA fluorochromes were incorporated distally to fluorescently monitor polymer removal. To begin AND NP synthesis, ligand particles were shielded with an MMP2 (Gly-Pro-Leu-GlyVal-Arg-Gly) ${ }^{12,13}$ substrate-polymer and receptor particles were shielded with an MMP7 (Val-Pro-Leu-Ser-Leu-Thr-Met) ${ }^{14}$ substrate-polymer. Enzyme specificity for these sequences was assessed by monitoring TAMRA fluorescence increase in the presence of various enzymes. Specific enzyme-substrate pairs rapidly increased TAMRA fluorescence as cleaved substrate fluorochromes diffused away from the quenching iron oxide cores (Figure S1). Non-specific pairs, including human thrombin, factor Xa, and serum, showed little fluorescence increase. We next examined each enzyme's ability to actuate assembly of peptide-shielded particles in 
the presence of their unmodified cognate particles. Specific enzyme-substrate pairs rapidly catalyzed the formation of nano- and micro-assemblies when incubated with unmodified cognate NPs, while non-specific pairs negligibly affected population size (Figure S2). To investigate the relationship between peptide polymer removal and NP assembly, biotin-MMP2PEG enzyme reactions were quenched at various timepoints (Figure S3) and incubated with receptor NPs. Following removal of $\sim 50 \%$ of NP polymers, assembly begins, eventually revealing sufficient biotin to direct NP precipitation.

To create a logical AND NP system, MMP2-PEG ligand particles with MMP7-PEG receptor particles were combined. Here, in presence of either protease alone, assembly of NPs was prohibited by the PEG polymers remaining on the cognate particle. In the presence of both proteases, however, NP self-assembly began and the population hydrodynamic radius increased 5-fold over 3 hours in DLS. Further, nanoparticles were able to express AND logic in T2 relaxation changes, mapped using a 4.7T Bruker MRI. In the presence of both enzymes, $\mathrm{T} 2$ relaxation is enhanced by $\sim 30 \%$ as compared to samples with either enzyme alone or none (Figure 1b). This enhancement is comparable to published magnetic relaxation sensors ${ }^{1,5,6}$, and occurs at MMP2 concentrations that mimic tumor activity levels in vivo (4ug MMP2/ml $=221 \mathrm{U} / \mathrm{ml}$ vs. $435 \mathrm{U} / \mathrm{g}$ in vivo $^{13}$ ).

A second system was constructed to actuate assembly in the presence of either of two proteolytic inputs (Logical OR). Here, only the particles containing the ligand were masked with peptide-linked polymers. To allow either enzyme to actuate assembly, a tandem MMP2MMP7 peptide substrate was synthesized, containing both cleavage motifs in series (separated by an aminohexanioc acid spacer). Hydrodynamic radii increased more than 5-fold in the presence of either or both enzymes, indicating proper OR function (Figure 2b). Accordingly, OR NP T2 relaxation decreases $\sim 40 \%$ in the presence of either or both enzymes, as compared to samples with no enzyme (Figure 2b).

In conclusion, we have demonstrated the synthesis of NPs that use Boolean logic to simultaneously monitor multiple biological processes associated with tumorigenesis. In the future, we anticipate that logical NP switches may enable more informative imaging of neoplastic transformation in optically opaque samples both in vitro and in vivo. The modular design of these logical NP sensors can be applied to other enzymatic triggers, complimentary ligand/receptor pairs, or NP cores (semiconductor, plasmonic). Looking further, logical NP switches may enable specific localization of the processes underlying malignant transformation in vivo, as proteolytically-assembled beacons in sites of neoplastic inception. Such interstitial assembly may amplify the retention of particles (by mechanical entrapment in the tumor interstitium) and allow MRI visualization of diagnostic logic functions.

\section{Supplementary Material}

Refer to Web version on PubMed Central for supplementary material.

\section{Acknowledgement}

We thank Erkki Ruoslahti for helpful discussions and critical review of this work. Financial support from NIH (BRP: R01CA124427-01), NIH/ NCI (R01CA124427-01), Packard Fellowship (1999-1453), Whitaker Foundation Graduate Fellowship and NSF Graduate Fellowship (G.v.M.).

\section{References}

1. Perez JM, Josephson L, O'Loughlin T, Hogemann D, Weissleder R. Nature Biotechnology 2002;20 (8):816-820. 
2. Mirkin CA, Letsinger RL, Mucic RC, Storhoff JJ. Nature 1996;382(6592):607-609. [PubMed: 8757129]Elghanian R, Storhoff JJ, Mucic RC, Letsinger RL, Mirkin CA. Science 1997;277(5329): 1078-1081. [PubMed: 9262471]

3. Georganopoulou DG, Chang L, Nam JM, Thaxton CS, Mufson EJ, Klein WL, Mirkin CA. Proceedings of the National Academy of Sciences of the United States of America 2005;102(7):2273-2276. [PubMed: 15695586]

4. Perez JM, Simeone FJ, Tsourkas A, Josephson L, Weissleder R. Nano Letters 2004;4(1):119-122.

5. Perez JM, Simeone FJ, Saeki Y, Josephson L, Weissleder R. Journal of the American Chemical Society 2003;125(34):10192-10193. [PubMed: 12926940]

6. Harris TJ, von Maltzahn G, Derfus AM, Ruoslahti E, Bhatia SN. Angew Chem Int Ed Engl 2006;45 (19):3161-3165. [PubMed: 16642514]

7. Wang Z, Levy R, Fernig DG, Brust M. J Am Chem Soc 2006;128(7):2214-2215. [PubMed: 16478166] Zhao M, Josephson L, Tang Y, Weissleder R. Angewandte Chemie-International Edition 2003;42(12): $1375-1378$.

8. Chang C, Werb Z. Trends in Cell Biology 2001;11(11):S37-S43. [PubMed: 11684441]Edwards DR, Murphy G. Nature 1998;394(6693):527-528. [PubMed: 9707109]

9. Stearns ME, Wang M. Cancer Research 1993;53(4):878-883. [PubMed: 7679051]Talvensaari-Mattila A, Paakko P, Turpeenniemi-Hujanen T. British Journal of Cancer 2003;89(7):1270-1275. [PubMed: 14520459]Davidson B, Goldberg I, Kopolovic J, Lerner-Geva L, Gotlieb WH, Ben-Baruch G, Reich R. Gynecologic Oncology 1999;73(3):372-382. [PubMed: 10366463]Kanayama H, Yokota K, Kurokawa Y, Murakami Y, Nishitani M, Kagawa S. Cancer 1998;82(7):1359-1366. [PubMed: 9529029]Fang JM, Shing Y, Wiederschain D, Yan L, Butterfield C, Jackson G, Harper J,

Tamvakopoulos G, Moses MA. Proceedings of the National Academy of Sciences of the United States of America 2000;97(8):3884-3889. [PubMed: 10760260]Ratnikov BI, Deryugina EI, Strongin AY. Laboratory Investigation 2002;82(11):1583-1590. [PubMed: 12429818]Giannelli G, FalkMarzillier J, Schiraldi O, StetlerStevenson WG, Quaranta V. Science 1997;277(5323):225-228. [PubMed: 9211848]

10. Rudolph-Owen LA, Chan R, Muller WJ, Matrisian LM. Cancer Res 1998;58(23):5500-5506. [PubMed: 9850086]Hulboy DL, Gautam S, Fingleton B, Matrisian LM. Oncol Rep 2004;12(1):1317. [PubMed: 15201952]

11. Pacheco MM, Mourao M, Mantovani EB, Nishimoto IN, Brentani MM. Clin Exp Metastasis 1998;16 (7):577-585. [PubMed: 9932604]

12. Seltzer JL, Akers KT, Weingarten H, Grant GA, McCourt DW, Eisen AZ. J Biol Chem 1990;265 (33):20409-20413. [PubMed: 2173706]

13. Bremer C, Tung CH, Weissleder R. Nature Medicine 2001;7(6):743-748.

14. Turk BE, Huang LL, Piro ET, Cantley LC. Nat Biotechnol 2001;19(7):661-667. [PubMed: 11433279] 


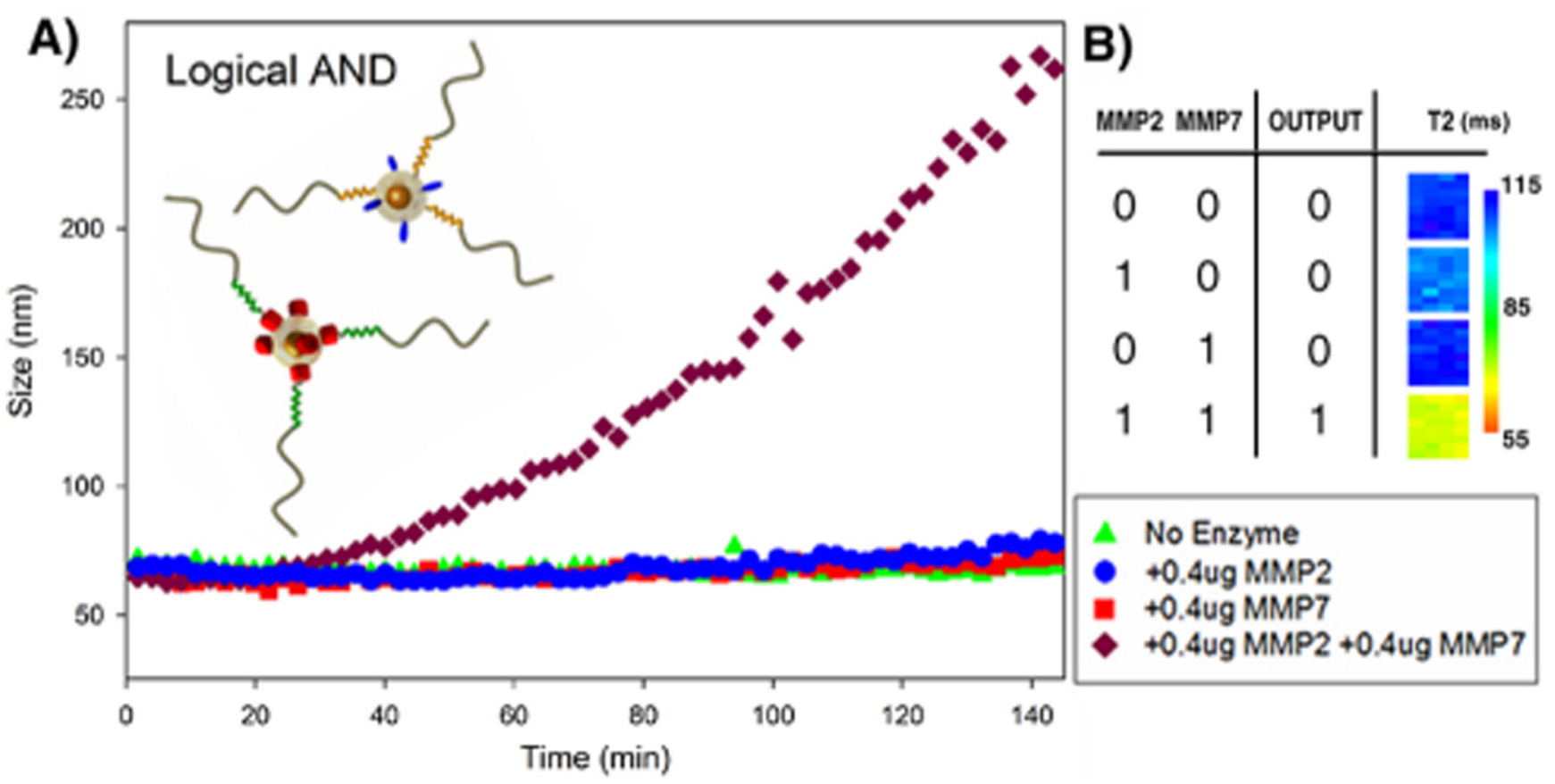

Figure 1.

Logical AND a) Hydrodynamic radius in dynamic light scattering is only increased in the presence of both MMP2 and MMP7; either or none is insufficient to actuate assembly (40ug $\mathrm{Fe} / \mathrm{ml}$ ). b) Assemblies express AND logic in MRI. T2 relaxation decreases $~ 30 \%$ in 3 hours following addition of MMP2 and MMP7, with nominal changes following addition of either enzyme alone (7.5ug $\mathrm{Fe} / \mathrm{ml})$. 


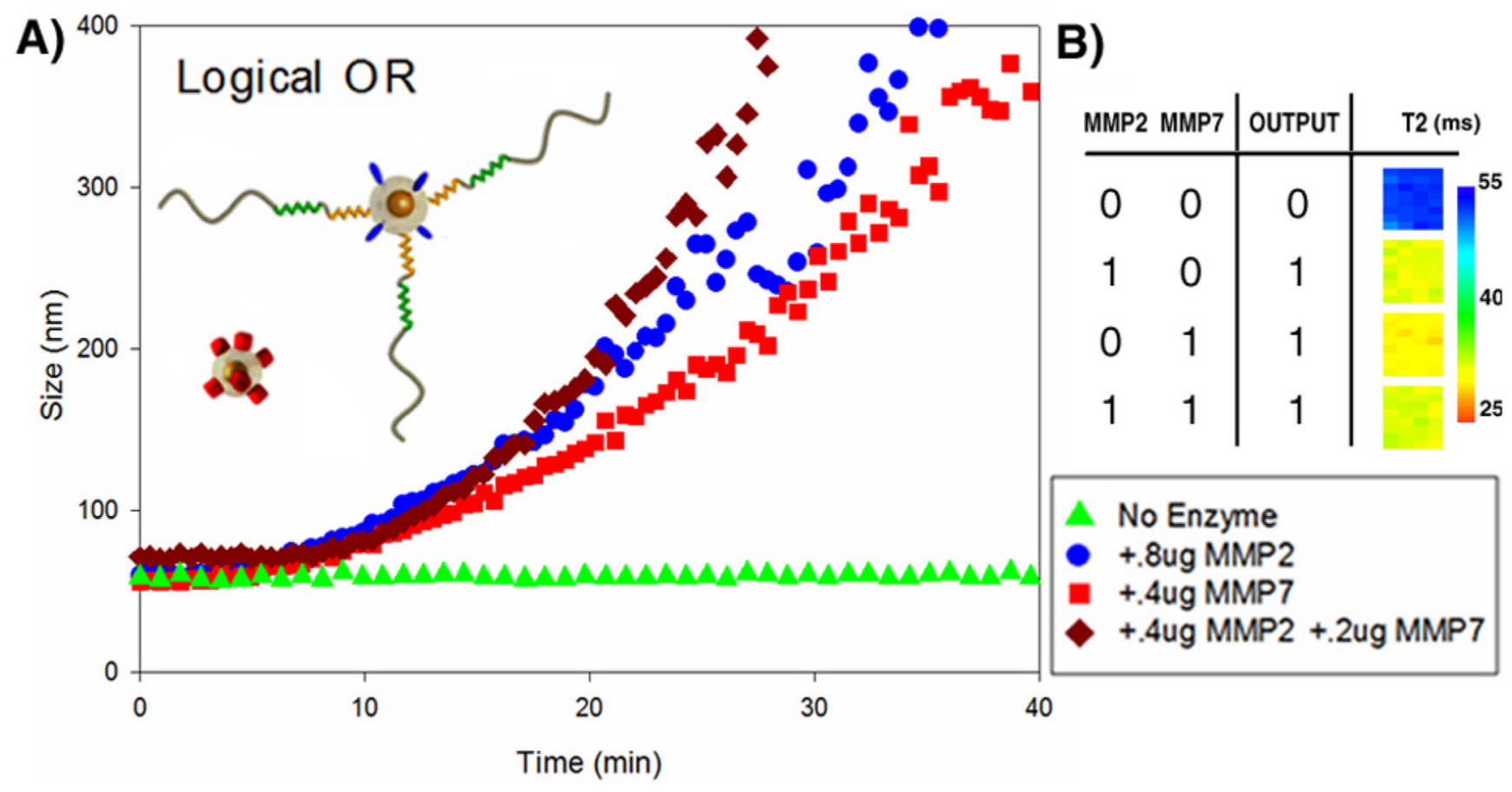

Figure 2.

Logical OR a) Population hydrodynamic radius is increased in the presence of either or both MMP2 and MMP7 (40ug/ml Fe). b) MRI visualization of logical function demonstrates 40\% enhancement in T2 relaxation in the presence of either MMP2 or MMP7 or both enzymes $(15 \mathrm{ug} / \mathrm{ml} \mathrm{Fe})$. 


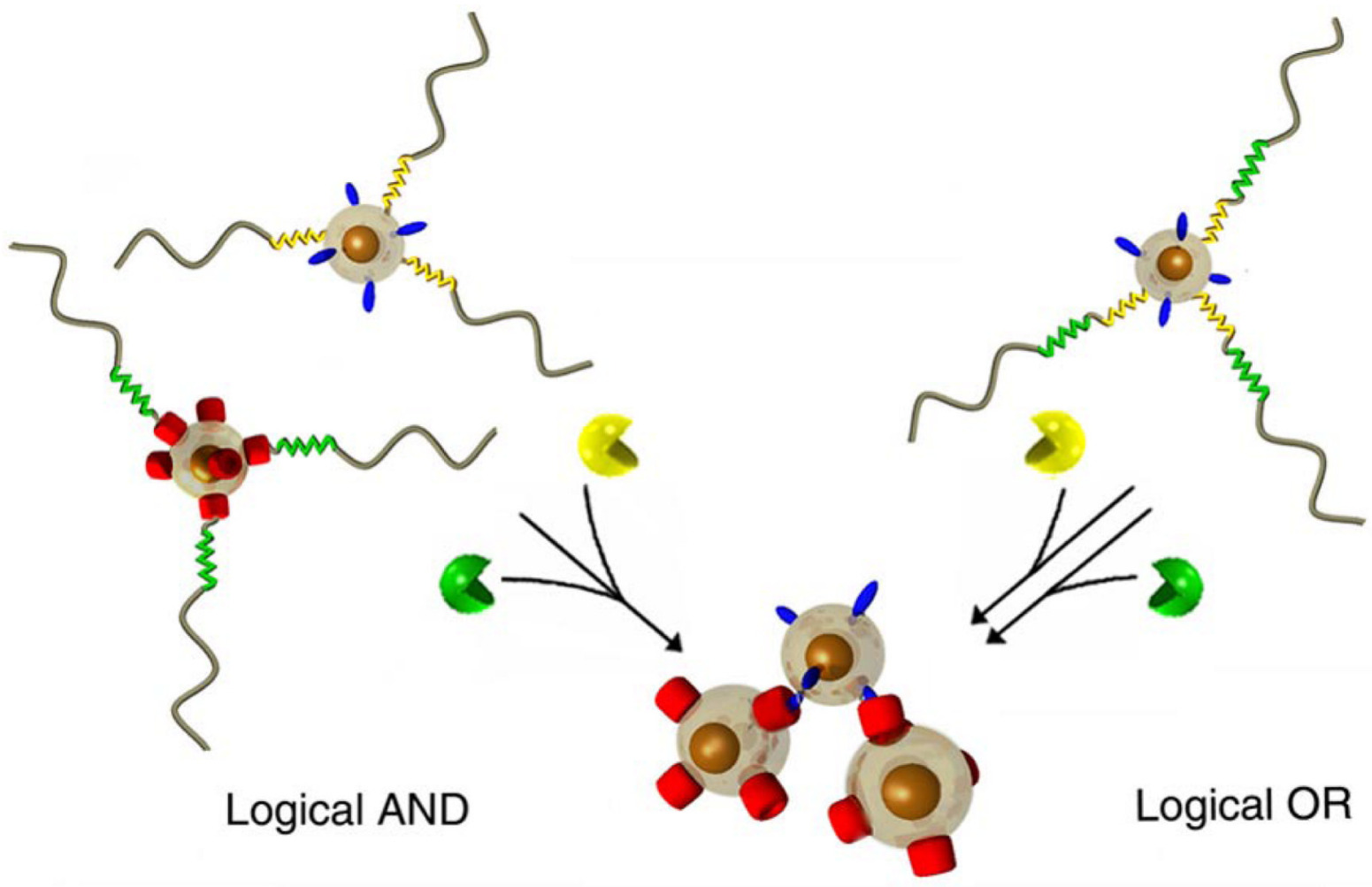

Avidin 1 Biotin MMP2 substrate MMP7 substrate

$\backsim$ Polyethylene glycol

Scheme 1.

Schematic Representation of Logical Nanoparticle Sensors. Self-Assembly is gated to occur in the presence of MMP2 and MMP7 (Logical AND) (Left) or in the presence of either or both proteases (Logical OR) (Right) by attachment of protease-removable polyethylene glycol polymers. 